\title{
SOOBRE DOIS NOVOS PARASITOS DE PEIXE DA COSTA DO PERU (CESTODA, TETRAPHYLLIDEA)'
}

\section{A. ARANDAS RÊGO e J. JULIO VICENTE N. IBÁNEZ HERRERA}

Instituto Oswaldo Cruz, Rio de Janeiro, Brasil Facultad de Ciências Biologicas Trujillo, Peru

(Com 3 estampas)

$\mathrm{Na}$ presente nota estudamos alguns cestódeos coletados de Sarda chilensis (Cuv.) peixe comum da costa do Pacífico, enviado por um de nós (N. I. H.) ; o material era constituído por vários espécimes de Phyllobothriidae e apenas três de Onchobothriidae, infelizmente todos comprimidos; não obstante aqui fazemos o seu estudo por se tratar de material de interêsse.

PHYLLOBOTHRIIDAE Braum, 1900

Anthobothrium peruanum sp. $\mathrm{n}$.

(Figs. 1-3)

Comprimento pelo menos $170 \mathrm{~mm}$; máxima largura do estróbilo $3,1 \mathrm{~mm}$; os proglotes imaturos, inclusive os que mostram apenas órgãos masculinos, são sempre mais largos do que longos; os segmentos que apresentam órgãos femininos amadurecidos mas sem apresentar ovos visíveis, medem $1,98 \mathrm{~mm}$ x $1,65 \mathrm{~mm}$; não encontramos proglotes com ovos ou o útero delineado, provàvelmente destacaram-se do estróbilo $\mathrm{e}$ foram perdidos durante a coleta dos parasitos.

Escólex grande, apresenta 4 botrídias pedunculadas, mais ou menos circulares, com margens crenuladas apresentando espessamentos de natureza muscular. O escólex com as botrídias em expansão mede $6,93 \mathrm{~mm}$ de diâmetro máximo, cada botrídia mede $2,64 \mathrm{~mm}-3,03 \mathrm{~mm}$ de diâmetro. Observam-se duas formas, uma com o escólex e botrídias

1 Recebido para publicação a 5 de julho de 1967.

Trabalho do Instituto Oswaldo Cruz (Divisão de Zoologia) e da Facultad de Ciencias Biologicas. 
maiores, a outra, com botrídias menores, não observamos no entanto diferenças morfológicas entre as duas. Não há myzorhynchus ou quaisquer ventosas accessórias. Em cada pedúnculo penetram dois canais excretores, um de cala dado, no interior das botrídias êles têm trajeto sinuoso que pode ser fàcilmente acompanhado. O pescoço é longo, mede $13 \mathrm{~mm}$ de comprimento e $1,5 \mathrm{~mm}$ de largura.

A genitália masculina, além de aparecer primeiramente é a única presente por grande número de proglotes, observam-se por grande extensão do estróbilo apenas proglotes com testículos e órgãos tubulares, não sendo observadaas nem glândulas vitelógenas nem ovário; nestes segmentos há cêrca de 280 testículos dispostos por todo o proglote, unindo-se na base e acima, mas sem ocupar a região mediana onde se notam órgãos tubulares em formação; nos segmentos mais posteriores, onde há órgãos femininos em maturação, contamos cêrca de 300 testículos distribuídos como acima explicado. A bôlsa do cirro mede $0,805 \mathrm{~mm} \mathrm{x}$ $0,175 \mathrm{~mm}$ nos proglotes maduros, essas medidas podem variar dentro de certos limites; no interior da bôlsa pode-se observar o cirro, sinuoso, armado de pequenos espinhos em tôda a extensão; fora da bôlsa seguese o canal deferente mui pouco sinuoso.

Os poros genitais abrem-se no $10^{\circ}$ têrço lateral, alternam irregularmente no estróbilo.

$\mathrm{O}$ ovário ocupa a base dos segmentos, não tem forma de $\mathrm{X}$ como referido em outras espécies, apresenta aspecto delicado, bilobado, formado por delgados lobos que partem da região mediana do órgão, mede $1,68 \mathrm{~mm}$ de largura. O vitelino é folicular, folículos pequenos, dispõemse nas partes laterais, um tanto afastados uns dos outros, são de difícil observação talvez devido à compressão do estróbilo. A vagina abre-se anteriormente à bôlsa do cirro, tem as paredes espêssas e é alargada em quase tôda a extensão; na região proximal parece formar delgado receptáculo seminal. Proglotes grávidos não vistos.

Não obtivemos bons cortes devido à compressão do estróbilo, mas nota-se que o sistema muscular é constituído por uma só camada de músculos longitudinais junto à região medular; notam-se também alguns feixes de músculos transversos limitando com a medula. O sistema excretor não foi bem compreendido ao que parece apresenta apenas o canal dorsal, não vimos o canal longitudinal ventral.

Tipos depositados na Coleção Helmintológica do Instituto Oswaldo Cruz sob os números 30.307 a-f e na Coleção da Facultad de Ciências Biológicas (parátipos).

Habitat - Intestino de Sarda chilensis (Cuv.).

Proveniência - Paita, Piura (Costa do Oceano Pacífico), Peru.

Discussão - Êstes espécimes apresentam como caracteres mais destacados, as botrídias grandes e pedunculadas, com os bordos crenulados, além do grande número de testículos e o ovário bilobado, com delicados prolongamentos.

Fizemos a discussão com as 20 espécies relacionadas por Yamaguti (1959), embora não tenhamos obtido algumas das descrições das espé- 
cies, muito especialmente o trabalho monográfico de SoUTHwELL (1925) sôbre os tetrafilídeos, por esta razão estabelecemos a espécie com certas reservas.

Não obstante, os nossos espécimes foram comparados com as espécies mais representativas de Anthobothrium; em nossa opinião $A$. peruanum $\mathrm{sp}$. $\mathrm{n}$. se distingue suficientemente das demais espécies do gênero não sòmente pelo aspecto e dimensões das botrídias (neste particular a espécie que mais se aproxima é $A$. cornucopia Beneden, 1850), mas principalmente pelo número de testículos (neste caracter a espécie mais próxima é $A$. pristis Woodland, 1934), mas dela difere por muitos outros caracteres morfológicos do escólex e estróbilo.

ONCHOBOTHRIIDAE Braun, 1900

\section{Acanthobothrium chilensis sp. $n$.}

(Figs. 4-7)

Comprimento pelo menos $40 \mathrm{~mm}$; máxima largura do estróbilo $1,5 \mathrm{~mm}$; os proglotes em maturação são mais ou menos retangulares; os do fim do estróbilo medem $1,71 \mathrm{~mm} \times 0,89 \mathrm{~mm}$ (são mais longos do que largos); o último segmento, que fàcilmente destaca-se, mede $2,1 \mathrm{~mm}$ de comprimento $\times 0,840 \mathrm{~mm}$ de largura.

O escólex mediu $0,990 \mathrm{~mm} \times 1,35 \mathrm{~mm}$ (mais largo do que longo), apresenta 4 botrídias alongadas no sentido longitudinal, não são pedunculadas, medem $0,840 \mathrm{~mm} \mathrm{x} 0,322 \mathrm{~mm}$, duas costae transversas dividem-nas em 3 lóculos, o lóculo anterior é maior do que os outros, são pouco musculosas e a fenda botridial é pouco perceptível em nossas preparações; cada botrídia apresenta um par de ganchos bifurcados, cujas extremidades têm dimensões pouco diferentes; comprimento dos ganchos (da extremidade do cabo à extremidade da maior lâmina) $0,220 \mathrm{~mm}-0,230 \mathrm{~mm}$; a extremidade mais longa mediu $0,133 \mathrm{~mm}$ e a menor $0,119 \mathrm{~mm}$; a máxima largura dos ganchos é $0,130 \mathrm{~mm}$; os ganchos têm os bordos espessos e com uma proeminência articular junto ao cabo. Anteriormente às botrídias e ganchos, notam-se ventosas accessórias, uma para cada botrídia. Das proximidades das botrídias partem fortes músculos longitudinais que podem ser acompanhados ao longo do pescoço. Pescoço bastante longo, mede 6,6 $\mathrm{mm}-9 \mathrm{~mm}$ de comprimento. Os poros genitais alternam irregularmente, abrem-se lateralmente, no têrço mediano dos segmentos.

Contamos 80-100 testículos por segmento, são fàcilmente observados nos segmentos em maturação, nos proglotes terminais a observação é dificultada pelo grande desenvolvimento dos órgãos femininos; êles são mais ou menos arredondados, medem $0,105 \mathrm{~mm} \times 0,070 \mathrm{~mm}$, dispõem-se por todo o proglote, separados em dois campos pelo útero e também limitados com o ovário. A bôlsa do cirro é piriforme, mede $0,350 \mathrm{~mm} \times 0,122 \mathrm{~mm}$ (no último segmento), no interior nota-se o cirro enrolado, espinhoso; num dos espécimes o cirro evertido mediu $0,315 \mathrm{~mm}$ de comprimento. O canal deferente apresenta nos segmentos terminais 
o máximo desenvolvimento, é muito dilatado contendo no interior espermatozóides flagelados, ocupa pois grande extensão na parte anterior do proglote.

O ovário não foi inteiramente compreendido quanto à forma e seus limites; nos anéis imaturos aparece como um U irregular, cuja extremidade do lado anti-poral atinge a região equatorial; nos últimos segmentos observam-se grossos folículos que atingem a bôlsa e os do lado oposto ultrapassam a linha equatorial dos segmentos. Tendo apenas 3 espécimes e infelizmente todos comprimidos não temos elementos para julgar esse grande alongamento da extremidade do ovário, isto é, se tratar-se-ia de caracter constante ou se devido à compressão sofrida pelos proglotes durante a fixação; no entanto parece-nos fora de dúvida que os folículos do lado anti-poral têm a tendência de atingir e mesmo ultrapassar a região mediana como pudemos acompanhar em todos os segmentos mesmo jovens, além disto, outras espécies do gênero mostram a mesma característica (embora não tão pronunciadamente), isto foi figurado por AleXANDER (1953) para A. holorhini e A. unilateralis. O vitelino é constituído por pequenos folículos lateralmente dispostos, pouco nítidos (talvez devido à compressão); a vagina abre-se anteriormente à bôlsa, é pouco dilatada, não pudemos observar receptáculo seminal, que provàvelmente existe. O útero, formado inicialmente por um tubo longitudinal, dilata-se nos últimos segmentos, formando divertículos pouco distintos; os ovos não foram vistos.

Material - tipo depositado na Coleção Helmintológica do Instituto Oswaldo Cruz, sob o número 30.308 a-c.

Habitat - Intestino de Sarda chilensis (Cuv.).

Proveniência - Paita, Piúra (Costa do Pacifico), Peru.

Considerações - Trata-se da primeira referência de parasitos dêste gênero na América do Sul, além disto também a primeira referência dêstes tetrafilídeos em peixes Teleósteos, pois segundo refere YamaguTi (1959) os Onchobothriidae ocorrem em Elasmobrânquios; não temos elementos para sugerir se tratar-se-ia de um parasitismo acidental.

Discussão - Acanthobothrium chilensis sp. $\mathrm{n}$. foi discutido com as descrições das espécies de Acanthobothrium van Beneden que pudemos obter; achamos mais próximas A. coronatum (Rudolphi, 1819) e A. holorhini Alexander, 1953, particularmente a primeira, cujos caracteres são tão aproximados que inicialmente pensamos ser idêntica à nossa espécie, a falta de mais abundante material, consideramos que a diferença fundamental entre elas reside no incomum alongamento do ovário em $A$. chilensis sp. n.; A. holorhini Alexander, 1953 possui ovário cuja forma lembra a nossa espécie e os ganchos são de iguais dimensões, difere no entanto pelas menores dimensões do estróbilo, escólex e botrídias e pelo menor número de testículos. A espécie qúe descrevemos é pois intermediária entre as duas espécies acima, aproximando-se muito mais de $A$. coronatum (Rudolphi, 1819). 


\section{REFERÊNCIAS BIBLIOGRÁFICAS}

Alexander, C. G., 1953, Five new species of Acanthobothrium (Cestoda: Tetraphyllidea) from southern California rays. J. Parasit., 39 (5) : 481-486, 15 figs.

Goldstein, R. J., 1964, Species of Acanthobothrium (Cestoda: Tetraphyllidea) from the gulf of Mexico, J. Parasit., 50 (5): 656-661, 12 figs.

Linton, E., 1908, Helminth fauna of Dry Tortugas. Carnegie Inst. Wash., 102 (1) : 1959-190, 11 pls.

Linton, E., 1916, Notes on two cestodes from the spotted sting-ray J. Parasit., 3 (1) : 35-37, 2 figs.

REES, G., 1943, The anatomy of Anthobothrium auriculatum (Rud.) (Cestoda) from Raja batis L. Parasitology, 35 (1-2): 1-70, 17 figs.

ReEs, G. \& Williams, H. H., 1965, The functional morphology of the scolex and genitalia of Acanthobothrium coronatum (Rud.) (Cestoda: Tetraphyllidea). Parasitology, 55 (5): 617-651, 43 figs., 3 pls.

WARdLE, R. A. \& McLeod, J. A., 1952, The Zoology of Tapeworms, 780 pp., 419 figs., Univ. Minnesota Press ed., Mineapolis.

Woodland, W. N. F., 1927, A revised classification of the Tetraphyllidean Cestoda, with descriptions of some Phyllobothriidae. Proc. Zool. Soc. London, 1927 (2): 519-548, 5 pls.

Woodland, W. N. F., 1934, On six new cestodes from Amazon fishes. Proc. Zool. Soc. London, 1934, Part I : 33-44, 2 pls.

Woodland, W. N. F., 1934, Some more remarkable cestodes from Amazon siluroid fish. Parasitology, 27 (2): 207-225, 5 pls.

Yamaguti, S., 1934, Studies on the helminth fauna of Japan. Part. 4. Cestodes of fishes. Jap. J. Zool., 6 (1) : 15-112, 187 figs.

YamaGuTI, S., 1952, Studies on the helminth fauna of Japan. Part 49. Cestodes of fishes, II. Acta Med. Okayama, 8 (1): 1-76, 22 pls.

Yamaguti, S., 1959, Systema Helminthum, 2, The Cestodes of Vertebrates: 1-626, 769-860, 70 pls., 584 figs. Interscience Publishers, Inc. ed., New York.

Young, R. T., 1954, Cestodes of sharks and rays in southern California. Proc. Helm. Soc. Wash., 21 (2) : 106-112, 8 figs. 


\section{ESTAMPA I}

Anthobothrium peruanum sp. $\mathrm{n}$.

Figura 1 - Escolex.

Figura 2 - Proglote maduro.

Figuras originais. 


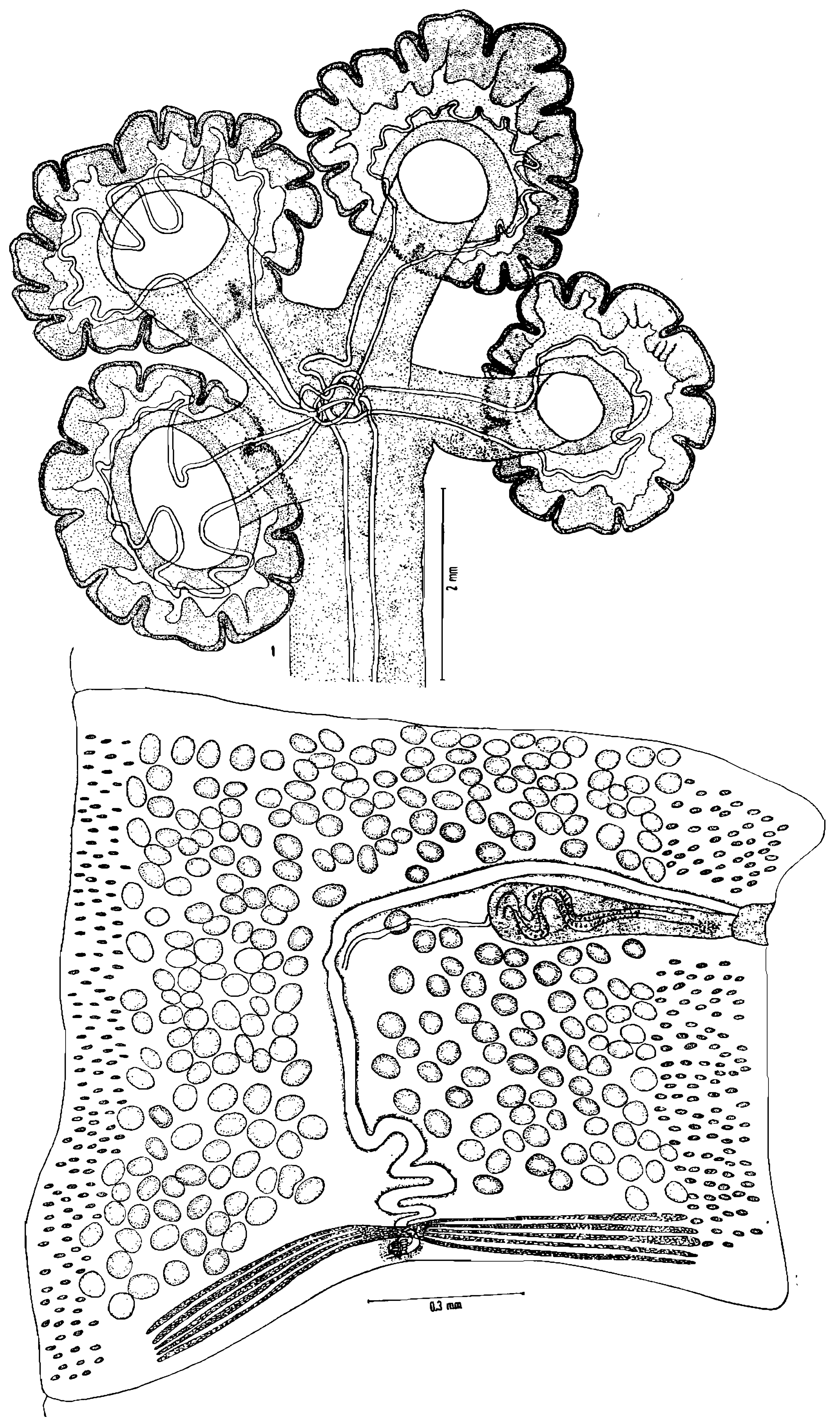

Rêgo, Vicente e Ibáñez: Parasitos de peixe do Peru 


\section{ESTAMPA II}

Acanthobothrium chilensis sp. n.

Figura 4 - Escolex.

Figura 5 - Proglote maduro.

Figuras originais. 

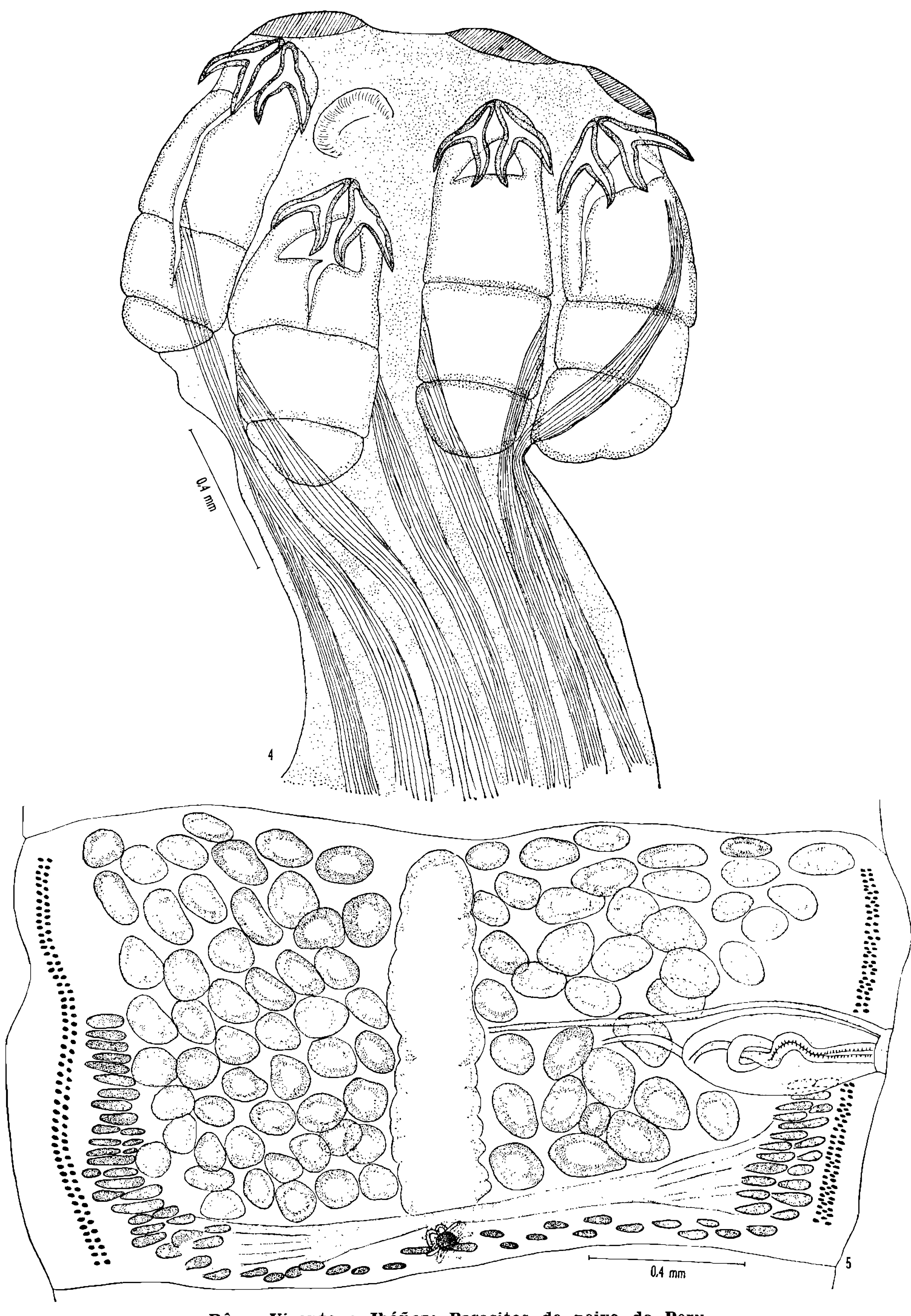

Rêgo, Vicente e Ibáñez: Parasitos de peixe do Peru 
ESTAMPA III

Anthobothrium peruanum sp. $\mathrm{n}$.

Figura 3 - Corte transversal de parte de proglote (ML: Musculatura longitudinal; T: Testículos; MT: Musculatura transversal).

Acanthobothrium chilensis sp. $\mathrm{n}$.

Figuras 6 e 7 - Ganchos em diferentes posições, na mesma escala. Figura 8 - Último proglote do estróbilo. 


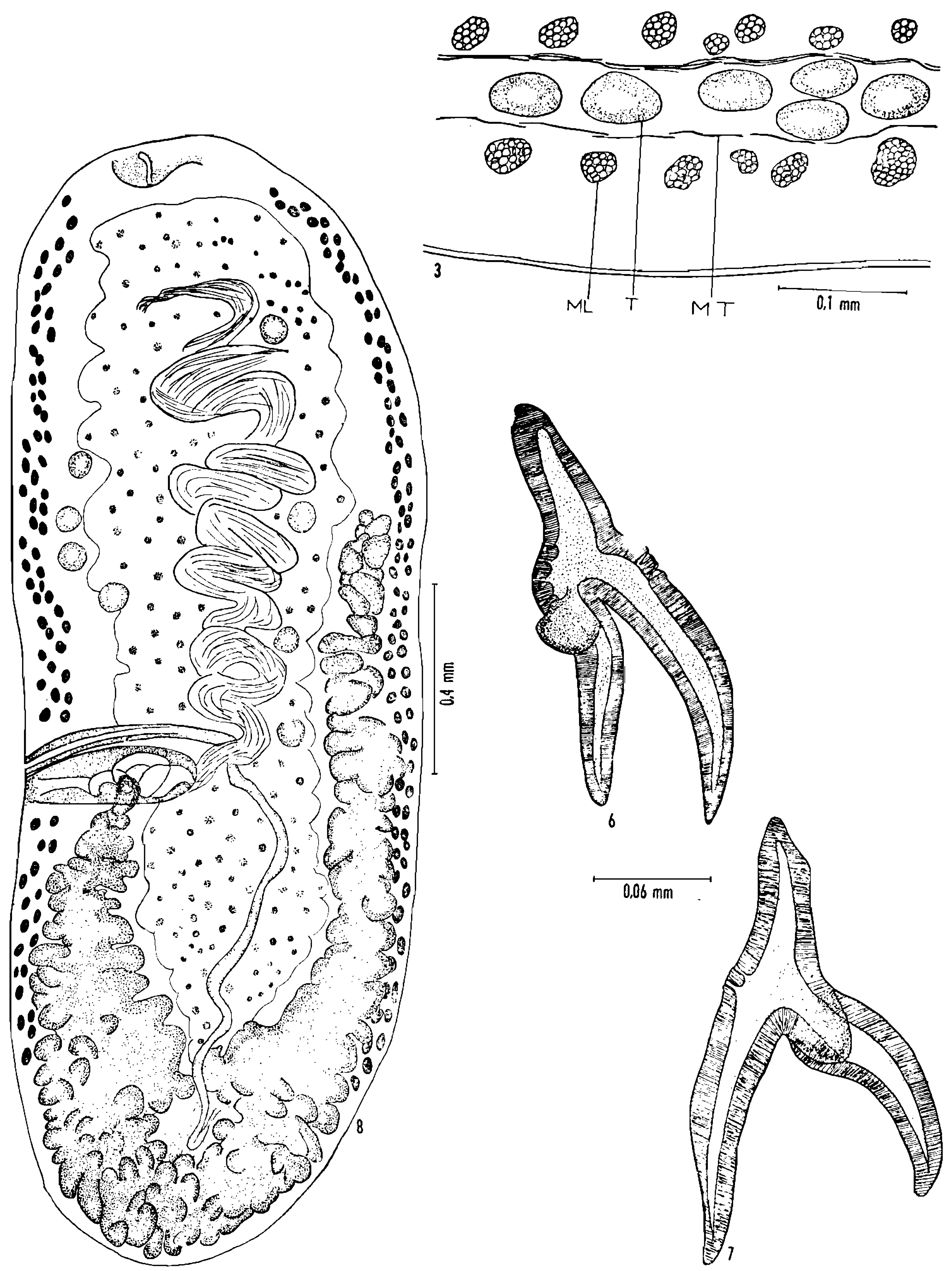

Rêgo, Vicente e Ibáñez: Parasitos de peixe do Peru 